\title{
Hour Times Nanogram per Milliliter per Milligram
}

National Cancer Institute

\section{Source}

National Cancer Institute. Hour Times Nanogram per Milliliter per Milligram. NCI

Thesaurus. Code C85627.

Hours times nanograms per milliliter per milligram. 